Service social

\title{
Le travail social à l'aube de 1990 : nouvelles tâches et remise en question des approches de formation
}

\section{Louis Favreau}

Volume 36, numéro 2-3, 1987

Approches intégrées

URI : https://id.erudit.org/iderudit/706374ar

DOI : https://doi.org/10.7202/706374ar

Aller au sommaire du numéro

Éditeur(s)

École de service social de l'Université Laval

ISSN

1708-1734 (numérique)

Découvrir la revue

Citer cet article

Favreau, L. (1987). Le travail social à l'aube de 1990 : nouvelles tâches et remise en question des approches de formation. Service social, 36(2-3), 478-491.

https://doi.org/10.7202/706374ar
Résumé de l'article

Le travail social a connu des remises en question en profondeur de sa place et de son rôle dans la société : une première crise, plus repérable dans les années 70 , a trait à l'identité ; on parle davantage de crise de légitimité dans les années 80.

Ayant situé le contexte des sociétés industrielles capitalistes avancées, l'auteur fait ressortir quelques pistes devenir et quelques tâches pour le travail social, tout en soulignant les impacts sur la formation. 
FAVReau, Louis, professeur au département des sciences humaines, Université du Québec à Hull.

\section{Le travail social à l'aube de 1990 : nouvelles tâches et remise en question des approches de formation}

\section{Louis Favreau}

Qu'observe-t-on lorsque l'on examine le métier et la profession de travailleur social ? Dans un certain nombre de milieux, à la faveur de la montée des mouvements sociaux, le travail social n'a-t-il pas connu une crise, c'est-à-dire une remise en question en profondeur de sa place et de son rôle dans la société ? Dans cette optique, je formulerai deux hypothèses, l'une liée au travail social tel que conçu et pratiqué dans les années 70 et l'autre, portant sur le travail social des années 80 . Par la suite, j'esquisserai quelques pistes d'avenir tout en identifiant les incidences sur la formation dans ce domaine.

\section{Le travail social aujourd'hui : crise d’identité et/ou crise de légitimité ? 1}

\section{Années 70 : crise d'identité ?}

Au cours des années 70 , de nouveaux et jeunes intervenants, se situant à la périphérie du travail social, commencent à le contester. Ils mènent une action axée sur la défense des droits sociaux : animateurs impliqués dans des conseils de développement social ou des conseils régionaux de développement, organisateurs communautaires de C.L.S.C., employés d'organismes communautaires financés par des sources religieuses ou par des Centraides... ou même, organisateurs communautaires devenus conseillers à la mobilisation, à l'information, à l'action politique ou à la formation dans des syndicats. 
Cette minorité s'engage dans un travail de soutien concret à des comités de citoyens, associations de locataires, organismes de défense d'assistés sociaux ou de protection du consommateur, garderies populaires et coopératives... Elle dispose de sources d'inspiration d'envergure internationale : dans un premier temps, elle a les yeux rivés sur l'organisation communautaire américaine d'orientation conflictuelle, type Saul Alinsky. ${ }^{2}$ Dans un second temps, elle part à la recherche de "la voie royale " de la transformation sociale et se met en quête d'un projet de société socialiste. Elle trouve inspiration dans l'approche de conscientisation développée par Paulo Freire en Amérique latine. ${ }^{3}$ Elle suit de près, du côté de l'Europe, les débouchés politiques à l'action sociale que développe la nouvelle gauche issue du mouvement de mai (mai 68) en France, ou de l'automne chaud italien (1969). ${ }^{4}$

Contesté à sa périphérie par cette minorité, le travail social l'est aussi de l'extérieur, et de façon assez virulente, par des mouvements essentiellement revendicateurs. Nous pensons, entre autres, aux premières occupations d'agences par des gens vivant de l'assistance sociale ou encore, à la pensée de gauche de cette époque concernant le rôle des travailleurs sociaux. Un texte assez marquant d'un collectif d'étudiants de l'Université de Montréal, paru au début de la décennie 70, en fournit un bel exemple : de leur point de vue, le travail social est au service de la classe dominante parce qu'il favorise la reproduction des rapports sociaux. Il exerce la "fonction perverse " d'agent d'intégration et il ne trouve sa raison d'être que dans le système capitaliste. Lorsque celui-ci sera aboli, le travailleur social le sera aussi. ${ }^{5}$ Figure de proue opposée à cet agent d'intégration: le militant, véritable agent de changement. Cette vision de la société est héritée du marxisme, ou plutôt d'un certain marxisme. Question angoissante qui en déstabilise plus d'un, à l'époque : de qui le travailleur social est-il le principal allié ? De l'État et des classes dominantes ou des classes populaires, des mouvements sociaux et de ses militants?

Contexte de la période : un État-providence en expansion et un État provincial en passe de devenir, peut-être, un État national ; mouvements sociaux à l'offensive, et jonction des uns avec les autres, mouvements populaire et étudiant, syndical et national réunis. D'où une place assez large à l'expérimentation et aux débats tous azimuts en dehors des institutions traditionnelles du service social, expérimentation d'ailleurs partiellement encouragée par l'État. Période pendant laquelle un nouveau secteur public émerge (les C.L.S.C.), tandis qu'un secteur communautaire autonome et radicalement différent de celui institué par les paroisses fait peu à peu son apparition dans nombre de quartiers populaires des grands centres urbains, tout comme dans les régions rurales éloignées. 
En somme, dans les années 70 , aux yeux d'une bonne partie des militants et militantes des mouvements sociaux, $y$ compris les organisateurs communautaires, le travail social semble avoir de moins en moins de pertinence pour répondre adéquatement aux problèmes de l'heure. Plutôt que de s'inscrire à l'intérieur du réseau traditionnel (la Corporation des travailleurs sociaux professionnels...), les organisateurs communautaires lutteront pour obtenir leur reconnaissance à partir du syndicalisme. Ils feront reconnaître leurs rôles et fonctions dans la convention collective des employés du secteur public, ${ }^{6}$ ce qui consacre en même temps leur indifférence à l'égard de la Corporation des travailleurs sociaux professionnels, qui d'ailleurs le leur rend bien.

\section{Années 80 : crise de légitimité ?}

Les années 80 prennent une toute autre allure: la crise d'identité s'est en partie résorbée. L'expérience a aussi permis de relativiser cette analyse trop sommaire et trop simpliste des années 70 . On sait mieux aujourd'hui la nature contradictoire de l'État, ses politiques inconsistantes, ses mesures et pratiques souvent incohérentes et donc la marge de manœuvre dont peut, à certaines conditions, disposer le travailleur social, même employé de l'État :

"L'appareil étatique n'est pas une machine bien huilée, au contrôle efficace, supervisée par les laquais du capitalisme. Les politiques sont inconsistantes et contradictoires car elles reflètent la nature compétitive de l'économie capitaliste et la crise qui la déchire" (Fleetwood et Lambert, 1982: 53). ${ }^{7}$

Mais une autre crise prend le relais, crise de légitimité cette fois. Au tournant des années 80 , à droite comme à gauche, on remet en question le travail social : du côté de l'État, du patronat et des politiciens, le travail social est jugé coûter trop cher pour ce qu'il est utile. Les C.L.S.C. ne sont-ils pas un luxe $?^{8}$ De l'autre côté, des usagers pensent de plus en plus, l'hypercentralisation aidant, que le travailleur social n'est qu'un fonctionnaire loin du monde et des problèmes réels. À leurs yeux, les centres de Travail-Québec sont remplis de "boubou-macoutes", selon l'expression des organismes de défense des assistés sociaux, et les Centres de services sociaux, bureaucratisés comme jamais auparavant.

Contesté à droite par le courant conservateur qui évoque le spectre de la crise financière ("Il faut dégraisser l'État ") et donc de l'impossibilité de répondre à toutes les demandes, le travail social est également critiqué à gauche par les milieux communautaires et alternatifs qui diagnostiquent un État bureaucratisé et en dérive technocratique. Bref, 
aux yeux de l'opinion sociale, un secteur public lourdaud rend de mauvais services alors qu'en dépit des contraintes de tous ordres, un secteur communautaire réussit à offrir un service de première ligne de qualité. À l'expert et au fonctionnaire éloigné des gens, on oppose le droit des usagers à de nouveaux services, à des services de qualité et à des ressources légères plus près des communautés locales. La question angoissante posée dans les années 70 est devenue celle-ci : comment le travailleur social peut-il faire son boulot sans perdre de vue le droit des usagers de participer aux décisions qui les concernent ? 9

On perçoit mieux aujourd'hui que l'État-providence, en partie issu des luttes du mouvement ouvrier et populaire, a certes été une réponse à certains besoins sociaux. Jamais on ne se départirait de l'assurancehospitalisation, de l'assurance-automobile, de l'assurance-chômage... D'où bien sûr, le refus des coupures de tout acabit.

Mais, car il y a un mais, ce mode particulier de réponse aux besoins sociaux comporte un prix à payer : le secteur public, règle générale, est centralisé et technocratisé. Incidences sur le travail social : 1) il se situe, de façon dominante, à l'intérieur de grandes structures des affaires sociales ; 2) il est éclaté et sur-spécialisé car la division du travail s'y est amplifiée ; 3) il consacre aussi la fin du monopole du travail social comme seule profession du domaine social car d'autres intervenants sont apparus et se sont multipliés :

"Les frontières entre les différentes professions sociales sont devenues de plus en plus fluctuantes. La catégorisation stricte de ce qui relève de l'action sociale, de l'action socio-éducative, de l'action culturelle, de certaines formes d'action économique paraît de plus en plus absurde. " 10

On pourrait donc, en schématisant, caractériser les deux périodes de la façon suivante:

Années 70

Années 80

Contexte:

État-providence en développement État-providence en crise

Caractérisation de l'État par les acteurs : État centralisé (bureaucratie État au service de la classe dominante .... et technocratie)

Situation du travailleur social:

Crise d'identité Crise de légitimité

Mouvement sociaux:

Défense de droits sociaux et action Développement de services politique socialiste et conscientisante alternatifs et nouveaux mouvements (paix) 


\title{
Lignes de force du travail social aujourd'hui
}

\author{
Les nouvelles trames de fond \\ des sociétés industrielles capitalistes avancées
}

Tenter d'identifier les défis qui se posent au travail social à l'aube de 1990, c'est d'abord chercher à débusquer les nouvelles trames de fond des sociétés contemporaines. Ces nouvelles assises nous renvoient notamment à l'informatique, aux biotechnologies etc. Jusqu'à preuve du contraire, nous sommes par là même conduits à la perte, plutôt qu'à la création d'emplois. Et encore, à la perte d'emplois dans l'industrie traditionnelle (textile, vêtement, mais aussi acier, fabrication de pièces destinées à des appareils électroniques...) qui dérive du nord vers le sud, des pays industrialisés vers les pays du tiers monde. L'évolution sociologique de l'économie et de l'emploi aujourd'hui pose le problème d'une société à deux vitesses, non seulement d'une société duale mais d'un dualisme à multiples faces: ${ }^{11}$ grandes entreprises versus petites entreprises, travail stable versus travail précaire, secteur tertiaire majoritaire versus secteur primaire et secondaire privé en déclin, hommes d'âge moyen versus hommes jeunes ou âgés, et finalement, hommes versus femmes sur le marché du travail.

La perte d'emplois, comme résultat de l'entrée sauvage des nouvelles technologies, et la segmentation de plus en plus marquée du marché du travail, s'accompagnent de certaines mutations aux plans culturel et idéologique. Primo, les solutions étatiques et les idéologies englobantes ont perdu, faut-il le redire, leur pouvoir de séduction; secundo, la transformation du travail devient de plus en plus marquée par la remise en cause de l'organisation du travail, de la qualité de vie au travail, du temps de travail, de la façon de travailler, de la valeur accordée au travail et, disons-le tout net, par l'apport décisif des femmes en la matière. Leur entrée massive sur le marché du travail depuis 20 ans, et surtout leur persistance à y demeurer malgré l'arrivée des enfants (en France, tout comme au Québec d'ailleurs, près de $2 / 3$ des femmes qui ont une activité professionnelle sont des mères de famille) ont contribué pour beaucoup à favoriser la mutation du travail et à renouveler les organisations liées à cette réalité, c'est-à-dire les syndicats. ${ }^{12}$ Cela pourrait signifier, pour les nouvelles générations surtout, un accroissement du temps libre, une possibilité beaucoup plus réelle de partager les tâches domestiques et d'éducation et, de façon plus globale, d'améliorer la qualité de vie. Cette évolution économique et culturelle provoque la révision des politiques sociales héritées de la période keynésienne, et de bon nombre de pratiques initiées par les mouvements sociaux. 


\section{Du côté des politiques sociales}

La conjoncture générale est marquée par la progression rampante de la société duale et du travail précaire. En même temps, la nouvelle conjoncture des politiques sociales, liée à la crise de l'économie et de l'État-providence, met sur l'avant-scène le temps de travail (de ceux qui ont un emploi) et l'emploi (pour ceux qui n'en ont pas). La période actuelle pose surtout le problème d'une réarticulation du social et de l'économique que l'État-providence avait soudés.

Une période de croissance lente ou nulle implique un chômage massif et l'exclusion sociale de couches de plus en plus importantes de la population. Dans ces conditions, les gouvernements sont amenés :

1) à affaiblir le secteur public par de nouvelles politiques de privatisation ;

2) à se désengager de certains secteurs dits sociaux, tels l'éducation populaire, l'information communautaire, le logement social, et à privilégier de nouvelles formes de création d'emplois (soutien à la très petite entreprise (TPE) et à la PME, puis secondairement, soutien à des coopératives de travail et à des corporations de développement économique communautaire) ;

3) à développer une politique de sous-traitance du secteur communautaire, en laissant aux organismes volontaires le fardeau du service tout en s'assurant d'un moindre coût global. ${ }^{13}$ II en coûte moins cher de financer des groupes populaires et communautaires qui reposent principalement sur des énergies bénévoles, que de dégager les ressources suffisantes à l'intérieur du réseau public (cas notamment des maisons d'hébergement pour femmes battues, de maisons de jeunes...);

4) à accentuer le planning et le contrôle des services sociaux de première ligne. N'assiste-t-on pas, dans bon nombre de C.L.S.C., au rétrécissement de la marge de manœuvre des intervenants communautaires dont les fonctions principales deviennent de plus en plus de faire pénétrer dans les collectivités locales des programmes et des services, prédéterminés par le M.S.S.S., au détriment de l'élaboration de programmes locaux, issus des collectivités elles-mêmes ? ${ }^{14}$

\section{Du côté des mouvements sociaux}

Cette évolution économique et culturelle a aussi influencé les mouvements sociaux : 
1) ils se sont retrouvés sur la défensive, tout particulièrement le mouvement syndical et les organisations populaires ;

2) leur projet social a été déstabilisé par un climat fait d'incertitudes (après beaucoup de certitudes!) et dans un contexte qui ne valorise plus le politique et l'idéologique mais bien davantage l'économie et l'action à court terme.

\section{Les nouvelles assises du travail social des années à venir, à l'aube de 1990}

Mais nous n'en sommes plus au repli des mouvements sociaux. À partir d'une enquête sur le mouvement populaire et communautaire de la région métropolitaine, ${ }^{15}$ nous concluons qu'après une période de morosité, de crise et de burn-out des intervenants sociaux, les mouvements connaissent pour la plupart une période de reprise. Leur capacité de rebondissement s'exprime comme suit :

\section{Le développement de nouveaux créneaux}

1) la jonction d'un courant féministe et des organisations populaires et communautaires, jonction qui a permis notamment l'éclosion de plusieurs des centres de femmes, puis, leur regroupement, I'R. des centres de femmes; 16

2) la jonction entre le social et l'économique dans ce qu'il est convenu d'appeler de plus en plus l'économie sociale : coopératives de travail, entreprises alternatives, corporations de développement économique communautaire en milieu urbain (C.D.E.C.), groupes de soutien à des jeunes ou à des femmes qui veulent entrer ou retourner sur le marché du travail ;

3) le travail commun entre deux composantes du secteur communautaire qui, hier encore s'ignoraient, voire même étaient en conflit et en concurrence : la composante "populaire » (groupes de revendications sur les conditions de vie) et la " composante communautaire" (groupes centrés sur l'entraide);

4) l'interaction dynamique entre les groupes qui luttent pour le désarmement et la paix, et les "vieux " mouvements que sont les syndicats et les organisations populaires et communautaires;

5) une ouverture de plus en plus active des groupes populaires aux communautés ethniques, à la lutte contre la discrimination dont elles peuvent être victimes, à l'établissement de passerelles avec les Québécois de vieille souche. 


\section{L'expression de nouvelles sensibilités}

Militer autrement, travailler autrement et vivre autrement deviennent aujourd'hui le nouveau leitmotiv. Changement d'époque et de mentalité : on assiste à la désaffection des formes plus traditionnelles d'action politique. Degré zéro de la propagande idéologique. Refus de la boursouflure militante et de l'idéologie réponse-à-tout. On mise davantage sur la qualité de vie, au travail, dans le quartier, à la maison. On veut que l'action entreprise débouche avant l'an 2000. Pragmatisme de bon aloi. L'investissement personnel est d'ailleurs multiforme: activités communautaires ou syndicales, voire même politiques (la politique municipale surtout) mais aussi, vie de couple, éducation des enfants et activités culturelles. Finalement, la faim dans le monde et la survie de la planète viennent toucher plus profondément que la question nationale ou un projet socialiste de société.

\section{La reprise de la réflexion théorique}

À la différence des années 70 marquées par une lecture des problèmes sociaux trop exclusivement politique et en fonction des classes, on ne peut plus se contenter d'un schéma d'analyse classes dominantes/classes dominées sans qu'interviennent les rapports hommesfemmes, les rapports entre générations et le rapport à la culture, c'està-dire la transformation des modes de vie. En ce sens, on peut parler d'un début de renouvellement théorique. Mais, certains l'affirment plus clairement que d'autres, il faut dépoussiérer l'analyse marxiste. En réalité, quand on refait l'inventaire de la littérature marxiste des quinze dernières années, on s'aperçoit qu'elle permet d'identifier un certain nombre de blocages au changement social mais ne peut discerner les nouveaux leviers d'action. Brièvement, l'approche marxiste classique, celle de Louis Althusser, de Nicos Poulantzas ou de Martha Harnecker ne convient pas tellement à la situation, parce qu'elle considère les mouvements sociaux comme des institutions, à la limite pouvant être classées sans difficulté parmi les appareils idéologiques de l'État. Axe central de cette approche : la fonction hégémonique des classes dominantes sur les classes ouvrières et populaires. Résultat: des études toujours centrées sur les contradictions inhérentes au capitalisme, avec peu ou pas de place accordée aux organisations de femmes, syndicales, populaires ou autres, sinon comme organisations utiles à la mobilisation initiale, mobilisation vite relayée par le parti d'avant-garde.

R. Hyman, sociologue marxiste anglais, et spécialiste du syndicalisme et des relations industrielles, affirme avec raison, et avec des airs 
d'autocritique : " combien est modeste la contribution marxiste spécifique à la compréhension du syndicalisme ". ${ }^{17}$ La clé du problème réside dans la considération suivante :

"Dans le contexte marxiste, seule la théorie du parti peut faire le pont entre les structures et les pratiques... Donc par définition, le concept de mouvement social comme agent de changement est tout à fait impensable dans la théorie marxiste. ${ }^{18}$

Est alors apparue une autre approche sociologique, dite des mouvements sociaux, plus complémentaire de l'approche marxiste qu'elle ne paraît, mais longtemps perçue comme antagoniste. Sont représentatifs de ce courant Alain Touraine en France, mais aussi Claus Offe en Allemagne, Alberto Melucci en Italie, pour qui la préoccupation centrale concerne les leviers d'action, la capacité spécifique des acteurs et des mouvements sociaux à travailler à la transformation sociale. J'ajouterais que le renouvellement du marxisme par les économistes dits de la régulation comme A. Liepietz, R. Boyer, A. Granou, B. Billaudot et Y. Barou, D. Leclerc, aide aussi à sortir des ornières althussériennes. ${ }^{19}$

Ce bref retour critique sur le marxisme ne peut manquer de remettre en cause les approches de formation en travail social. Pourquoi par exemple se limiter à une seule approche en organisation communautaire comme on le fait dans certaines universités? Pourquoi avoir boudé pendant plus d'une décennie le "grassroots community organisation " aux États-Unis?

\section{De quelques tâches actuelles du travail social}

Après cette digression sur le marxisme, revenons plus directement à l'objet premier de cette réflexion. Le travail social des années à venir fait face à un problème énorme de centralisation des services sociaux et, par voie de conséquence, de centralisation du syndicalisme dans ce secteur. Il faut, croyons-nous, s'interroger sur la pertinence et la validité de la décentralisation des services, malgré les risques pour les travailleurs qui y sont engagés (risques de retour aux inégalités salariales, aux disparités des conditions de travail...). Par contre, devant la dérive technocratique dans laquelle les affaires sociales sont engagées, il faut miser sur la proximité des services et sur les avantages qui en découlent pour les usagers.

En deuxième lieu, il est clair que dans la conjoncture actuelle, l'émergence et le développement du secteur communautaire remettent en cause la place dévolue au secteur public. Il faut sortir du modèle caractérisé par le monopole du secteur public sur les services sociaux, 
autant que du modèle défini par la sous-traitance confiée au secteur communautaire. Il faut s'engager dans la perspective d'un projet de société moins étatiste sans pour autant tomber dans le panneau de la privatisation classique. II faut accréditer des alternatives sociales et économiques concrètes. À ce propos, les pistes de solution émergent à peine de leur période d'incubation.

Enfin, troisième pièce du puzzle, si on ne veut pas, dans la foulée de la récession économique et du chômage massif, s'enliser dans la séparation de l'économique et du social, il faut investir le terrain économique, travailler à bâtir une économie sociale. L'évolution de l'opinion publique est capitale en cette matière : le chômage est-il, à ses yeux, un problème stratégique?

Décentralisation des services de l'État, développement d'un secteur communautaire autonome et vigoureux, démarrage d'une véritable économie sociale : telles sont, en résumé, quelques-unes des tâches auxquelles les travailleurs sociaux devront s'attaquer. Il convient cependant de les inscrire dans une stratégie renouvelée de changement social.

\section{Une stratégie qui s'attaque au problème de l'emploi et du désarmement}

Élargissons l'horizon en introduisant quelques éléments de prospective et de perspective. D'abord au plan de l'économie en général, ce qui vient tout de suite à l'esprit, c'est le plein emploi. Stratégie du souhaitable, mais utopique si elle ne s'accompagne pas de changements significatifs au niveau de l'organisation du travail, du temps de travail, des modes de vie. En d'autres termes, lutter pour la réduction et le partage du temps de travail, investir dans l'organisation d'une économie sociale, contribuer au désarmement et à la paix en stimulant, entre autres, la reconversion industrielle, défendre l'environnement, voilà quelques pistes incontournables de transformation sociale.

\section{Une stratégie d'élargissement des droits démocratiques dans la société}

Bon nombre d'organisations vivent la période actuelle, non pas comme un repli et une défaite, mais bien comme une accumulation de forces : chacun prend le temps d'échanger, de confronter et de vérifier, d'où là préférence pour les réseaux (versus les organisations), pour les débats (versus l'inscription dans un projet précis), pour l'expérimentation 
à petite échelle (versus les grandes mobilisations dans la rue). Si ce type d'action collective ne mène pas nécessairement à une société radicalement différente, elle favorise à coup sûr l'extension de la démocratie, l'élargissement des espaces d'expression de liberté, bref « des lieux de respiration sociale " et, en bout de ligne, jette les bases pertinentes pour un projet social transformateur.

Dans cette perspective, il nous semble que le travail social a beaucoup d'avenir : en dépit de sa crise de légitimité et même d'une crise d'identité, il devra, pour ce faire, répondre aux besoins nouveaux découlant de l'allongement de la durée de vie (activités sociales, communautaires et culturelles du troisième âge), de l'accès des femmes au travail salarié (organisation sociale en fonction des jeunes enfants: garderies...), et aux besoins nouveaux découlant de la protection de l'environnement, de la transformation de l'habitat... ${ }^{20}$

\section{Les incidences sur la formation en travail social}

Des sociétés de plus en plus complexes et en transformation rapide amènent donc le travail social, à l'aube de 1990, à ne plus s'exercer de la même façon. De nouvelles tâches pointent à l'horizon. Tout cela n'est évidemment pas sans incidence sur l'enseignement, ou de façon plus générale, sur la formation en travail social.

Primo, l'exigence d'une meilleure formation générale se fait sentir en psychologie, en sociologie et en sciences politiques, tout comme en économie et en histoire. Les étudiants se présentent sur un marché du travail qui exigera d'eux plus de polyvalence et une combinaison adéquate de formation générale et de formation spécialisée. Ils risquent d'avoir plusieurs "jobs" et employeurs différents, contrairement à la génération d'il y a quinze ans qui a pu bénéficier de l'expansion du secteur public en allant chercher la sécurité d'emploi comme corollaire.

Secundo, la formation spécialisée est certes nécessaire mais doit être orientée vers l'ensemble de la communauté locale. Le travail social est devenu trop sectoriel et son enseignement a subi les effets de ce découpage. Les problèmes sociaux sont compartimentés, résultat d'un monde du travail bureaucratisé et d'une planification effectuée par des gestionnaires. En ce sens, il faut valoriser une formation qui met en relief la connaissance des politiques sociales mais qui souligne également l'existence des communautés locales et des mouvements sociaux, de même que l'influence politique de ces organisations et leur apport à la démocratie et à l'économie. 
Tertio, la formation en travail social doit emboîter le pas en suivant de près l'évolution et le développement de nouveaux champs d'intervention, sortir du jardin québécois et du pré français pour réfléchir plus et mieux à toutes les questions qui nous préoccupent, miser sur la méthode comparative, notamment avec les États-Unis et l'Angleterre. Faut-il rappeler ici que Saul Alinsky a laissé des héritiers ; malheureusement, au Québec, nous en sommes encore à parler de lui comme si nous étions à la fin des années $60 .{ }^{21}$ De plus, il est loin d'être le seul auteur américain pertinent à notre situation.

Quarto, valoriser le travail social de coopération et de solidarité internationales, ce qui, jusqu'à maintenant, a été très peu fait : échange de professeurs entre universités du Québec et de pays du tiers monde, intensification de séminaires de recherche sur des thèmes de coopération et de solidarité internationales (développement communautaire comparé pays industrialisés/pays du tiers monde...), voire même des stages d'étudiants.

\section{Conclusion}

Le capital de protestation politique dans la rue (accompagnée d'un débat idéologique très virulent sur les rapports à l'État, le réformisme...) s'est certes effiloché, laissant plus d'un intervenant communautaire sur la brèche. Cette perte a été compensée, ce qui n'est pas encore suffisamment visible, par une vitalité socio-culturelle (groupes, services et réseaux nouveaux) et socio-économique (coopératives de travail, C.D.E.C., entreprises alternatives...) qui ne se dément pas. Cette vitalité n'est pas étrangère, bien au contraire, au renouvellement des pratiques auxquelles un certain nombre de travailleurs sociaux se sont associés ces dernières années. Cette voie me semble prometteuse. La formation en travail social ne doit-elle pas emboîter le pas ou mieux encore, participer activement à ce renouvellement ?

\section{Notes et références}

1 C'est un article du secrétaire général de la Fédération nationale des syndicats de services de santé et de services sociaux (C.F.D.T.) qui m'a mis sur cette piste : "Le travail social n'est pas en retard d'une crise", revue Économie et Humanisme, $n^{\circ} 286$, nov.-déc. 1985. Le présent texte s'inspire largement d'une communication faite à l'ouverture du colloque Techniques de travail social organisé par le Regroupement québécois des enseignants-es d'assistance sociale (CEGEP) tenu en juin 1987 à Montréal. 
2. Voir à ce propos, Alinsky, Saul, Le manuel de l'animateur social, Seuil/Point, Paris, 1976.

3 Voir Freire P., Pégagogie des opprimés, Maspero, Paris, 1974.

4 Voir à ce sujet Hamon H. et Rotman P., Génération : les années de rêve, Seuil, 1987 et Génération: les années de poudre, Seuil, 1988.

5 Noyau Service social, Le service social... instrument d'une classe ?, C.A.P. StJacques et Maisonneuve, Montréal, 1973. Mentionnons ici que tout un courant sociologique (la sociologie dite de la reproduction), vient alimenter ce type d'approche. Voir, parmi d'autres, les analyses de P. Bourdieu sur l'école, ou celles de M. Foucault sur le travail social, notamment dans Surveiller et punir.

${ }^{6}$ La convention collective des syndicats des affaires sociales stipule en effet qu'un organisateur communautaire est une " personne qui fait l'identification et l'analyse des besoins de la population avec les groupes concernés. Conçoit, coordonne et actualise des programmes d'organisation communautaire afin de répondre aux besoins du milieu et de promouvoir son développement. Agit comme personne-ressource auprès des groupes" (article 112 de la convention collective des syndicats affiliés à la FAS-CSN). On y retrouve également une définition du même ordre pour le travailleur communautaire.

7 Propos de Fleetwood, M. et Lambert J., Bringing Socialism Home: Theory and Practice for a Radical Community Action, dans Craig, G. Derricourt N. et Loney, M., Community Work and the State, Londres, 1982. Propos traduits et rapportés par la Revue internationale d'action communautaire: Craig, G., "L'action communautaire et l'État au Royaume Uni ", RIAC, no 17/57, Montréal, 1987: 170 .

8 Voir à ce propos l'article délirant de Dominique Demers dans la revue L'Actualité "A-t-on besoin des CLSC? ", Janvier 87 : 40-44.

9 Voir l'article de J.T. Godbout, Le scandale du CSSMM, La Presse, 27-28 juillet 87 , p. B 3. Godbout mentionne que l'on fait « comme si le scandale du CSSMM n'était qu'un incident regrettable, une bavure dans un système par ailleurs à l'abri de tout soupçon. C'est faux... ". Puis il explique que " cette irresponsabilité des cadres, cette insensibilité à l'urgence et à la gravité des problèmes, cet éloignement de la clientèle sont plus répandus qu'on ne le pense... "Tout cela trouve son explication dans l'approche technocratique, "celle du "clients come last", dans l'absence de représentation directe des dits clients dans l'organisation... " Pour une analyse institutionnelle critique de l'ensemble des services sociaux, voir son livre : La démocratie des usagers, Montréal, Boréal, 1987. Et pour une analyse en termes de classes et de mouvements sociaux à partir des CLSC, voir Bélanger, Paul R. et Lévesque, B., La bureaucratie contre la participation, Département de sociologie, UQAM, 1986.

10 Voir Thierry, M., Malaise dans le travail social ?, revue Économie et Humanisme, \# 286, nov.-déc. 1985 : 9.

11 Voir à ce propos Debouzy, M., Travail et travailleurs aux États-Unis, Paris, éd. La Découverte, 1984: 46-65 et Rosanvallon. P., "Crise et décomposition de la classe ouvrière" dans Crise et avenir de la classe ouvrière, Paris, Seuil/ politique \# 101, 1979: 21-38.

12 Voir Maruani, M., Les syndicats à l'épreuve du féminisme, Paris, Syros, 1979. Les données québécoises du recensement de 1986 indiquent que le taux de 
participation des femmes ayant des enfants d'âge pré-scolaire est de $60.8 \%$ (Le Devoir, 2/3/88).

13 Voir à ce sujet Bélanger P.-R. et Lévesque B., "Les nouveaux enjeux du mouvement populaire québécois", revue Relations, Montréal, mai 1985 : 120-123.

14 Voir à ce propos le rapport Brunet, Rapport du comité de réflexion et d'analyse des services dispensés par les CLSC, Gouvernement du Québec, MSSS, 10 mars 1987. Signalons cependant que le rapport de la commission Rochon prend la contrepartie de cette orientation (Rochon: 522).

15 Cette recherche sera publiée en 1988, Favreau Louis, Mouvement populaire et intervention communautaire (1960-1988) : continuités et ruptures. Voir aussi Corporation de développement communautaire des Bois-Francs, Les actes du colloque provincial sur le développement communautaire, tenu à Victoriaville les 16, 17 et 18 octobre 1986, Victoriaville, 1987.

${ }^{16}$ Ce n'est pas une coquille: L'R. pour le Regroupement, pour l'aire, pour l'ère... des femmes.

17 Hyman, R., "La théorie des relations industrielles: une analyse matérialiste", revue Sociologie du Travail, 4, 1979: 435. Voir aussi du même auteur, Marxism and the Sociology of Trade-Unionism, London, Pluto Press, 1971.

18 Castells, M., The city and the grassroots, Berkeley and Los Angeles, University of California Press, 1983: 299.

19 Pour une excellente synthèse du courant dit de la régulation en France, voir L'état des sciences sociales en France, Paris, Ed. La Découverte, 1986 : 223-229. Voir aussi quelques productions de ce courant notamment Granou, A., Baron, Y. et Billaudot B., Croissance et crise, Paris, Maspero/PCM, 1979; Clerc, D., Lipietz A. et al., La crise, Paris, Syros, 1983; Boyer, R., Capitalismes fin de siècle, Paris, PUF, 1986.

20 C'est dans ce sens que s'oriente la réflexion de certains syndicalistes. Voir E. Maire, Les nouvelles frontières du syndicalisme, Paris, Syros, 1987.

21 Voir à ce sujet Boyte H., The Backyard Revolution (Understanding the New Citizen Movement), Philadelphia, Temple University Press, 1981. 